\title{
Case Report: The Effect of Neurofeedback Therapy on Reducing Symptoms Associated with Attention Deficit Hyperactivity Disorder: A Case Series Study
}

Mostafa Deilami ${ }^{1}$, Asghar Jahandideh ${ }^{1}$, Yousef Kazemnejad ${ }^{1}$, Yousef Fakour ${ }^{1}$, Shiva Alipoor ${ }^{2}$, Fatemeh Rabiee ${ }^{3}$, Ghazal Saadat Pournesaie ${ }^{3}$, Rosemarie Noot Heidari ${ }^{4}$, Seyed Aliasghar Mosavi ${ }^{5}$

1. Deputy of Research and Technology, Ministry of Health and Medical Education, Tehran, Iran.

2. Department of Educational Psychology, Islamic Azad University, Saveh Branch, Saveh, Iran.

3. Department of General Psychology, Islamic Azad University, Tonekabon Branch, Tonekabon, Iran

4. Center for Development of Research \& Technology, Deputy of Research \& Technology, Ministry of Health and Medical Education, Tehran, Iran.

5. Department of Ophthalmology, Bina Eye Hospital Research Center, Tehran, Iran.

Citation: Deilami, M., Jahandideh, A., Kazemnejad, Y., Fakour, Y., Alipoor, Sh., Rabiee, F., et al. (2016). The effect of neurofeedback therapy on reducing symptoms associated with attention deficit hyperactivity disorder: A case series study. Basic and Clinical Neuroscience, 7(2), 167-171. http://dx.doi.org/10.15412/J.BCN.03070211

http://dx.doi.org/10.15412/J.BCN.03070211

Article info:

Received: 17 May 2015

First Revision: 23 June 2015

Accepted: 19 September 2015
Key Words:

Attention deficit, Das-Naglieri cognitive assessment system test, Hyperactivity disorder, Neurofeedback

\begin{abstract}
A B S T RA C T
Introduction: This study aimed to evaluate the effectiveness of neurofeedback on attention deficit hyperactivity disorder.

Methods: This is a quasi-experimental study without a control group. The study population included all children aged 5 to 12 years old affected with attention deficit hyperactivity disorders in Tehran, Iran who were referred to psychiatric clinics and given the diagnosis. The sample included 12 children with attention deficit hyperactivity disorder who were selected based on their availability (non-random sampling). They received 30 sessions of neurofeedback treatment, 2 times per week. Before and after neurofeedback training, the children were evaluated and compared with the use of cognitive assessment system test. Data were analyzed using dependent T-test.
\end{abstract}

Results: The total mean score for pretest was 88.81 while the total mean score for the post tes was 82.23 . The mean in pretest for attention hyperactivity disorder was higher than the mean in the post test. Moreover, The difference of pretest and post test scores of children affected with learning disorder associated with $\mathrm{ADHD}$ was calculated that showed significant $(\mathrm{P}=0.003)$.

Conclusion: Neurofeedback is effective in the improvement of attention deficit hyperactivity disorder.

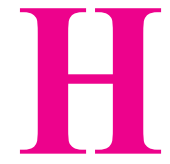

\section{Introduction}

yperactivity is a familiar term, especially for parents and teachers. Hyperactive Children are in constant motion, fidget and squirm in their seat, dash around, tap with their fingers, push others, loss their control for no reason. They often have difficulty in concentrating on organizing and completing a task or learning something new. When these behaviors get severe and persistent, they will be

* Corresponding Author:

Seyed Aliasghar Mosavi, MD

Address: Department of Ophthalmology, Bina Eye Hospital Research Center, Tehran, Iran

Tel: +98 (21) 22605915

E-mail: a.a.mosavi@gmail.com 
eligible to be diagnosed with Attention Deficit Hyperactivity Disorder (ADHD) (Davison \& Neale, 2000). ADHD is a common psychiatric disorder that affects approximately $3 \%-5 \%$ of school-aged children (American Psychiatric Association, 2000). Study of Moradi et al. (2008) on 722 elementary pupils estimated the prevalence of this disorder at approximately $12.5 \%$. Gender, type of delivery, sibling's birth order, and mother's education were not positively related to this disorder (Moradi et al., 2008). Diagnosis of this disorder depends on the therapist's expertise and has no precise laboratory test (Moradi et al., 2011).

The most obvious difference between the electrical activities of the brain in children with ADHD and those of normal children is seen in the frontal and central locations, which are associated with lack of arousal and decreased brain activity (Loo \& Barkley, 2005). Brain waves are classified in 4 different categories based on their frequency. These 4 categories are arranged in the order of their frequency as follows: delta (1 to $3 \mathrm{~Hz}$ ), theta (4 to $7 \mathrm{~Hz}$ ), alpha (8 to $13 \mathrm{~Hz}$ ), and beta (14 to $30 \mathrm{~Hz}$ ). Delta waves are seen when a person is in deep sleep and theta when the person is in a pre-sleep, semi-awake state. Alpha activity usually reaches to its maximum when the person is awake, relaxed, and calm. Beta waves are associated with focus and cognitive processing (Hammond, 2011). When a normal person is performing a significant task such as reading, doing simple arithmetic, or listening to a story, the electroencephalogram (EEG) waves usually change and the frequency and size of beta waves in the frontal areas (especially in the right frontal areas) increase.

However, people with hyperactivity disorder often function in the opposite direction and their EEG results demonstrate an increased slow theta wave frequency without any significant activity increase in the frontal region. Slow activity (theta waves) is a characteristic of disturbed mind, distraction, and decentralized thinking (Lubar, 2003). Children with ADHD demonstrate an increase activity in theta band and a decrease activity in beta band during resting period and in performing tasks (Wangler et al., 2011). Clarks and colleagues (2002) concluded that children with learning disorder associated with ADHD have increased proportion of theta wave, lower proportion of alpha wave and an increase in theta/alpha ratio compared to children having only ADHD (Clarke, Barry, McCarthy, \& Selikowitz, 2002).

One of the tools used in measuring the level of brain activity and make a record of the brain's spontaneous electrical activity over a short period of time is EEG. This procedure is safe and painless and its various func- tions can improve the brain's self control. Neurofeedback, also called neurotherapy or neurobiofeedback, is a type of biofeedback that uses realtime displays of EEG or hemoencephalography to illustrate brain activity and teach self-regulation (Masterpasqua \& Healey, 2003). Neurofeedback is a technique that focuses on helping people train themselves to directly affect brain function, i.e. neurofeedback works by providing individuals feedback of their brainwaves.

The goal of neurofeedback training is to modify abnormal EEG waves and accordingly improve individual's cognitive and behavioral performance. Therefore, an appropriate neurofeedback protocol can compensate the low theta/alpha ratio (Becerra et al., 2006). Neurofeedback significantly decreases the cognitive and behavioral symptoms of ADHD and its effectiveness is equal to drug therapy and or even a good alternative to stimulant drugs (Rossiter \& La Vaque, 1995). A series of case control studies regarding the effectiveness of biofeedback in the past 3 decades indicate that neurofeedback significantly improves attention, behavior control, increases cortical activity, and improves their score of IQ test and academic achievements (Leins et al., 2007).

Despite the favorable results of the application of positive conditioning by strengthening the electrophysiological activity (Lubar \& Shouse, 1976), few controlled studies have been conducted to determine the usefulness of neurofeedback. Most reports regarding the effect of neurofeedback were in the form of case studies and reported the patient's functioning in terms of intelligence and analysis of academic performance and behavioral rating scales before and after neurofeedback. Majority of the case studies (Alhambra, Fowler, \& Alhambra, 1995; Lubar, Swartwood, Swartwood, \& O’Donnell, 1995; Lubar, Swartwood, Swartwood, \& Timmermann, 1995; Thompson \& Thompson, 1998) yielded positive results; however, more controlled studies are needed to determine the efficacy of this treatment (Nash, 2000).

\section{Materials and Methods}

In this study, the dependent variable, attention deficit hyperactivity disorder, was measured before and after implementation of neurofeedback training with the use of cognitive assessment system (CAS) test. Because we had no control group, the pretest and post test design was implemented only in the experimental group which made it a quasi-experimental design. Study population included all children with ADHD referred to the psychiatric clinics in Tehran, Iran. Out of them, 12 children were qualified the inclusion criteria and were selected 
based on availability (non-random sampling). Although in a quasi-experimental research the sample size must not be less than 15 , in our case we had to opt for 12 subjects due to the lack of samples. Children aged 5-12 years old were given test to assess ADHD.

\subsection{Research tools}

\subsubsection{Cognitive assessment system test}

In this test, 4 scales of planning, attention, simultaneous, and successive cognitive processes were assessed. This test is designed for children and adolescents ranging from 5 to 17 years. Four cognitive processes in CAS (Najafi, Sadeghi, Molazade, Goodarzi, \& Taghavi, 2010). were coordinated with Luria's 3 structural unit protocols:

1. Processing based on Luria's first structural unit: brain stem, midbrain and the brain;

2. Simultaneous and successive processing based on Luria's second structural unit: occipital, temporal, and parietal lobes;

3. Planning based on Luria's third structural unit: the frontal lobe, especially the prefrontal lobe in the same direction.

Results of the study conducted on 1600 children showed that CAS scores and the scores of (Wood'sCook's, 2004 and revised WJ-RIII achievement test, 2010) were correlated having a correlation coefficient of $0.35-0.64$ and a reliability test of 80-90.

\subsection{Implementation method}

Neurofeedback training was implemented 2 times per week to a total of 30 sessions to all participants and each session lasted an hour. Comprehensive evaluation of the treatment process on the $5^{\text {th }}, 10^{\text {th }}$, and $15^{\text {th }}$ sessions were undertaken as well as psychological assessment sessions on the 10 th, $20^{\text {th }}$, and $30^{\text {th }}$ sessions. Before and after implementation of neurofeedback, CAS test was conducted on the participants to obtain the pretest and post test scores.

\section{Results}

In order to assess the effect of neurofeedback on children with ADHD, the pupils' score on the pretest and post test were compared. Table 1 shows the mean and standard deviation scores of ADHD assessment in the study group. According to the results, the total mean score for pretest is 88.81 while the total mean score for the post test is 82.23 . Therefore, we can conclude that the mean in pretest for attention hyperactivity disorder is higher than the mean in the post test.

To evaluate effect of neurofeedback, the differences between pretest and post test scores were calculated and then dependent $t$ test was conducted. The purpose of conducting the dependent $t$ test is to determine whether a difference exists between the pretest and post test score after the intervention. The results are shown in Table 2.

\section{Discussion}

The aim of this present study was to assess the effect of neurofeedback on children with ADHD. The obtained

Table 1. Mean and standard deviation of attention deficit disorder scores.

\begin{tabular}{|c|c|c|c|c|}
\hline \multirow{2}{*}{ ADHD } & Mean & SD & Mean & Pre-test \\
\cline { 2 - 5 } & Post-test & Post-test & 83 & 14.16 \\
\hline Planning & 69 & 14.15 & 94.9 & 16.21 \\
\hline Simultaneous processing & 85.5 & 17.16 & 101.5 & 90.57 \\
\hline Attention & 82 & 12.21 & 13.95 & 10.31 \\
\hline Sequential processing & 89.36 & 13.25 & 88.81 & 13.21 \\
\hline Total score & 82.23 & & NEUR:SCIENCE
\end{tabular}

Table 2. Analysis of dependent $t$ test on the pretest and post test scores of children affected with learning disorder associated with ADHD.

\begin{tabular}{|c|c|c|c|c|c|c|}
\hline & P-value & Mean & SD & Mean standard & df \\
\hline $\begin{array}{c}\text { ADHD pretest } \\
\text { and post test }\end{array}$ & 0.003 & -8.4 & 6.59 & 2.05 & -4.38 \\
\hline
\end{tabular}

NEUR:SCIENCE 
results indicate that neurofeedback training is an effective method on improving the symptoms of ADHD. The results of this study are consistent with findings of the previous studies conducted which indicated that neurofeedback can reduce cognitive and behavioral symptoms of ADHD when it is implemented for 15 weeks, 2 sessions per week (Fox, Tharp, \& Fox 2005).

One reason to explain these findings is the regulatory feature of neurofeedback on theta waves that are associated with distraction, inattentiveness, daydreaming, and anxiety (Chabot \& Serfontein, 1996). With the help of this regulatory feature, an improvement in the signs and symptoms of ADHD can be achieved. In this regard, (L'evesque \& colleagues, 2006) believed that in children with ADHD, neurofeedback can normalize the brain waves of these children leading to an improvement in their symptoms. In their study, neurofeedback training was conducted on children for $13 \frac{1}{2}$ weeks ( 3 sessions per week). Training sessions were divided into 2 parts of 20 sessions each: In the first 20 sessions the group was trained to increase the activity of beta bands and in the second 20 sessions the group was trained to decrease the activity of theta waves. After the training sessions, the differences between pretest and post test scores on the effects of neurofeedback on the brainwaves (decrease and increase in theta bands) were analyzed. In a study conducted by Wangler and associates, 2011) on 94 children with ADHD disorder, similar results were obtained showing the effects of neurofeedback on this group of children .

In conclusion, neurofeedback was able to improve the selective attention of this children but no improvement was observed in passive attention related to computer work. Neurofeedback as a behavioral technique has an important role in the attention on internal changes along with strengthening and increasing focus and attention of these children in other fields. Due to the limited studies conducted in this regard, it is suggested that similar study with a control group and a larger sample in both genders be implemented in the future.

\section{Acknowledgements:}

The authors would like to express their gratitude to the personnel of the psychiatric clinics, parents and children, and to our colleagues who contributed so much in the success of this study.

\section{References}

Alhambra, M. A., Fowler, T. P., \& Alhambra, A. A. (1995). EEG biofeedback: A new treatment option for ADD/ADHD. Jour nal of Neurotherapy, 1(2), 39-43.

American Psychiatric Association. (2000). Diagnostic and statistical manual of mental disorders (revised 4thed.). Washington, D.C.: American Psychiatric Association.

Becerra, J., Fernandez, T., Harmony, T., Caballero, M. I., Garcia, F., Fernandez-Bouzas, A., et al. (2006). Follow-up study of learning-disabled children treated with neurofeedback or placebo. Clinical EEG and neuroscience, 37(3), 198-203.

Chabot, R. J., \& Serfontein, G. (1996). Quantitative electroencephalographic profiles of children with attention deficit disorder. Biological Psychiatry, 40(10), 951-963.

Clarke, A. R., Barry, R. J., McCarthy, R., \& Selikowitz, M. (2002). EEG analysis of children with attention-deficit/hyperactivity disorder and comorbid reading disabilities. Journal of Learning Disabilities, 35(3), 276-285.

Davison, G. C., \& Neale, J. M. (2000). Abnormal Psychology, Study Guide. Hoboken, N.J.: John Wiley and Sons.

Fox, D. J., Tharp, D. F., \& Fox, L. C. (2005). Neurofeedback: an alternative and efficacious treatment for attention deficit hy peractivity disorder. Applied Psychophysiology and Biofeedback, 30(4), 365-373.

Hammond, D. C. (2011). What is neurofeedback: An update Journal of Neurotherapy, 15(4), 305-336.

Leins, U., Goth, G., Hinterberger, T., Klinger, C., Rumpf, N., \& Strehl, U. (2007). Neurofeedback for children with ADHD: A comparison of SCP and Theta/Beta protocols. Applied Psychophysiology and Biofeedback, 32(2), 73-88.

Loo, S. K., \& Barkley, R. A. (2005). Clinical utility of EEG in attention deficit hyperactivity disorder. Applied neuropsychology, 12(2), 64-76.

Lubar, J. F. (2003). Neurofeedback for the management of attention deficits disorders. In M. S. Schwartz \& F. Andrasik (Eds.), Biofeedback: A Practitioner's Guide (3rd ed.) (pp. 409-437). New York: Guilford Press.

Lubar, J. F., \& Shouse, M. N. (1976). EEG and behavioral changes in a hyperkinetic child concurrent with training of the sensorimotor rhythm (SMR). Biofeedback and Self-regulation, 1(3), 293 306.

Lubar, J. F., Swartwood, M. O., Swartwood, J. N., \& O'Donnell, P. H. (1995). Evaluation of the effectiveness of EEG neurofeedback training for ADHD in a clinical setting as measured by changes in TOVA scores, behavioral ratings, and WISC-R performance. Biofeedback and self-regulation, 20(1), 83-99.

Lubar, J. F., Swartwood, M. O., Swartwood, J. N., \& Timmermann, D. L. (1995). Quantitative EEG and auditory event-related potentials in the evaluation of attention-deficit/hyperactivity disorder: Effects of methylphenidate and implications for neurofeedback training. Journal of Psychoeducational Assessment, 34, 143-160.

Masterpasqua, F., \& Healey, K. N. (2003). Neurofeedback in Psychological Practice. Professional Psychology: Research and Practice, 34(6), 652. 
Moradi, A., Khabar Khob, M., Agah, T., Javaheer Forooshzadeh, A., Rezvan, B., \& Haeri Kermani, S. (2008). [The prevalence of Attention Deficit Hyperactivity Disorder in elementary pupils in the academic year 2006-2007 in Nishapour (Persian)]. Journal of Gorgan University of Medical Sciences, 10(2),37-42.

Moradi, A., Pouladi, F., Pishva, N., Rezaei, B., Torshabi, M., \& Mehrjerdi, Z. A. (2011). Treatment of anxiety disorder with neurofeedback: case study. Procedia-Social and Behavioral Sciences, 30, 103-107.

Najafi, S., Sadeghi, V., Molazade, J., Goodarzi, M. A., \& Taghavi, M. R. (2010). Brain cognitive functions in normal, ADHD and RD (Reading disable) children (A comparative study). Procedia-Social and Behavioral Sciences, 5, 1849-1853.

Nash, J. K. (2000). Treatment of attention deficit hyperactivity disorder with neurotherapy. Clinical EEG and Neuroscience, 31(1), 30-37.

Rossiter, D. T. R., \& La Vaque, T. J. (1995). A comparison of EEG biofeedback and psychostimulants in treating attention deficit/hyperactivity disorders. Journal of Neurotherapy, 1(1), 48-59.

Thompson, L., \& Thompson, M. (1998). Neurofeedback combined with training in metacognitive strategies: effectiveness in students with ADD. Applied Psychophysiology and Biofeedback, 23(4), 243-263.

Wangler, S., Gevensleben, H., Albrecht, B., Studer, P., Rothenberger, A., Moll, G. H., et al., (2011). Neurofeedback in children with ADHD: specific event-related potential findings of a randomized controlled trial. Clinical Neurophysiology, 122(5), 942-950 